\title{
Análisis litológico para incremento de la tasa de perforación en la cuenca oriente Ecuador
}

\author{
Diego Ayala ${ }^{1 *}$, Alejandro Bastidas ${ }^{2}$, Marco Loaiza ${ }^{3}$, Silvia Ayala ${ }^{4}$ \\ ${ }^{1}$ Facultad de Física. Universidad de Barcelona, PO Box: 08028. Barcelona-España. \\ ${ }^{2}$ Petroamazonas EP, PO Box 17-05-04, Quito. Ecuador. \\ ${ }^{3}$ Enap-Sipetrol PO Box 17-01-2759, Quito. Ecuador. \\ ${ }^{4}$ Instituto Superior Tecnológico Cotopaxi, Latacunga-Ecuador. \\ Email: diego.ayala.t@gmail.com
}

\begin{abstract}
Resumen
Se evaluará la tasa de perforación y la trayectoria de los pozos en estudio a partir de la información litológica, los esfuerzos regionales y la estabilidad en la cara del pozo para adaptar las trayectorias a la litología regional de forma tal que se aproveche de las características de la misma para mejorar la perforabilidad e incrementar la tasa de penetración.

Este artículo expone lo desafiante que resulta alinear todas las variables geológicas y disponerlas de manera que permitan optimizar la perforación por cuanto existe un interés puntual dentro del reservorio desarrollado y planteado por los ingenieros de yacimientos, el mismo que en ocasiones impide aprovechar la orientación de los esfuerzos principales en la cara del pozo, así como los esfuerzos regionales de la zona perforada. Al establecer una relación entre la litología, las características geomecánicas, los problemas al perforar y la tasa de penetración de los pozos analizados se determinó aquellos parámetros que permitieron en cada sección del pozo incrementar la tasa de perforación como respuesta a una mejor adaptación de la geología regional.

El trabajo incluye sugerencias de las posibles direcciones que podría tomar un pozo basados en los diagramas polares de estabilidad lo que conjuntamente con una trayectoria perpendicular a los esfuerzos regionales propiciaría un incremento en la tasa de penetración.
\end{abstract}

Palabras clave: Tasa de penetración, litología, Optimización de la perforación.

\section{Lithological Analysis to Increase the Drilling Rate of Penetration in the Oriente Basin - Ecuador}

\begin{abstract}
The drilling rate and the trajectory of the wells under study will be evaluated based on the lithological information, regional efforts and stability on the face of the well to adapt the trajectories to the regional lithology, thus taking advantage of the characteristics in respect thereof to improve the drivability and increase the penetration rate.

This article exposes the challenge of aligning all the geological variables and arranging them in a way that optimizes drilling, as there is a specific interest within the reservoir developed and proposed by reservoir engineers, which sometimes prevents taking advantage of the orientation of the main efforts on the face of the well, as well as the regional efforts of the perforated area. When establishing a relationship between lithology, geomechanical characteristics, drilling problems and the penetration rate of the wells analyzed, parameters were determined that allowed each section of the well to increase the drilling rate in response to a better adaptation of the geology.
\end{abstract}

The paper includes suggestions of the possible directions that a well could take based on polar stability diagrams, which, together with a trajectory perpendicular to regional efforts, would lead to an increase in the penetration rate.

Keywords: ROP, Drilling optimization, Lithology.

Cita: Ayala, D., Bastidas, A., Loaiza, M. \& Ayala, S. (2019). Análisis litológico para incremento de la tasa de perforación en la cuenca oriente Ecuador. Revista Fuentes: El reventón energético, 17(1), 83-94. 


\section{Introducción}

El principal objetivo en la perforación de un pozo es el potencial beneficio económico en la comercialización de hidrocarburos. El costo total de la operación de perforación es el resultado de una interacción compleja de muchos factores. (Graham \& Muench, 1959; Ayala et al, 2017; Ortiz et al, 2018). En operaciones de perforación normalmente la complejidad de la perforabilidad de un pozo disminuye con la profundidad (Garnier \& Van Lingen, 1959; Montes et al, 2018). La complejidad en las operaciones de perforación se ha incrementado mucho conforme aumenta la complejidad de las localidades de nuevos reservorios y los problemas se presentan en una magnitud proporcional a dicha complejidad, esto puede afectar de forma crítica la estimación de los costos de perforación. (Saleem \& Tunio, 2011; Ayala et al, 2016; Campana et al, 2017). Los diferentes métodos de varias disciplinas en actividades de perforación que son utilizados hoy en día se enfocan en la construcción de un pozo seguro, con un bajo impacto ambiental y rentable. (Tuna, 2010; Ayala et al, 2011; Medina et al, 2013). Este estudio se enfocará en el mismo objetivo mediante el estudio del comportamiento de los parámetros de perforación en las arenas productoras $\mathrm{y}$ en las formaciones arcillosas (inestables), se contrastarán los datos registrados para determinar aquellos valores que permiten la tasa de penetración más alta y de esta forma establecer las constantes a dichos parámetros que obedecen a la litología propia del campo en estudio, sin desestimar otras variables el estudio se centrará en aquellas que son de carácter operativo (ROP, WOB, caudal y densidad del lodo) de esta forma se suman dos beneficios, disminuir el tiempo de operaciones y limitar la exposición de los fluidos de perforación ante formaciones inestables y arenas productoras.

Este análisis considera los esfuerzos principales $\left(\sigma_{H}, \sigma_{h}, \sigma_{v}\right)$ en la cara del pozo y los esfuerzos regionales (asociados a la orientación del anticlinal que alberga a las zonas productoras donde están ubicados los pozos) para brindar recomendaciones sobre la dirección más favorable para la trayectoria de los pozos guardando una mejor correspondencia con dichos esfuerzos (Portilla et al, 2012; Loaiza et al, 2018).

Obtener un diseño de pozo en el cual la dirección con la que se debe ingresar en las formaciones de interés coincida con los esfuerzos principales y los regionales puede ser desafiante, sin mencionar que el problema puede ser más complejo cuando en la zona se encuentra una alta densidad de pozos y las trayectorias tienen que ser diseñadas bajo ciertos parámetros en dirección para evitar colisiones entre pozos.

Otro factor a considerar es la diferente dureza de las formaciones perforadas la cuales provocan una deflexión en las rocas lo que eventualmente puede ocasionar un cambio en la dirección del pozo. Las causas geológicas que determinan que las formaciones sean blandas y duras se deben principalmente al grado de cementación. Las areniscas de cuarzo bien cementadas son más duras que las areniscas pobremente cementadas, las rocas formadas de calcita u otros carbonatos son suaves. La composición mineral también afecta la dureza de la roca. Las areniscas de cuarzo limpias son más duras que las arcosas (areniscas con feldespato) y las grises (areniscas que contienen mucha arcilla). (Rashidi \& Hareland, 2009).

La falta de información del cambio de dureza en las formaciones causa varios problemas durante la perforación, por ejemplo, una alteración en la dirección planificada o el desgaste prematuro de la broca que incrementa el tiempo de perforación por viajes no planificados. Todo lo anterior es contrario al objetivo de optimizar la perforación. Estos incidentes desafortunados pueden ocurrir en las paredes y el fondo del pozo o provocar un desgaste del equipo y eventualmente provocar la falla del mismo (Solberg, 2012).

Los 5 pozos seleccionados para el estudio se encuentran en la locación del campo $\mathrm{X}$, poseen igual tipo de geometría direccional, tienen como objetivo la misma arena productora (Hollín inferior), se utilizaron similares configuraciones de BHA y Broca lo que da uniformidad a la muestra y facilita la comparación entre si para determinar los parámetros de optimización.

\subsection{Estructura campo en estudio}

Se presenta un anticlinal elongado según el eje N-S; se ha observado fallas que alcanzan a Hollín y Napo Basal (Bady, Rivadeneira, \& Barragán, 2014), no se observan fallas importantes por encima de la parte basal de la formación Tena. Estas fallas corresponden generalmente a fallas normales que han controlado la sedimentación, antes del depósito del Cretácico, durante la fase de extensión de la cuenca. (Almeida \& Cárdenas, 2010). 


\subsection{Factores Geológicos que inciden en la perforación}

Las consideraciones en la formación como dureza, estructura y buzamiento o inclinación, son factores que influyen para que un pozo se salga de su trayectoria.

En la práctica existen varios factores que dificultan mantener perfectamente la verticalidad en un pozo. Un pequeño ángulo fuera de curso es aceptable, pero obviamente, entre más se aparte un pozo de su trayectoria planeada, más probable es que no llegue a la zona prevista en el objetivo. Esto es un error cuya corrección costaría mucho tiempo y dinero pues el pozo requerirá el uso de costosas herramientas de fondo para dirigirlo a su curso correcto, o bien tendrá que ser re-perforado con el fin de llegar al objetivo. (Hawker, Vogt, \& Robinson, 2001).

\subsubsection{Ambientes de depositación del campo en estudio}

En el campo se han identificado cuatro ambientes de depositación (fluvial, carbonatado y turbidítico) en las zonas de interés (Hollín, T, U y Basal Tena):

Hollín, La Arenisca Hollín se depositó en un ambiente fluvial evolucionando a un ambiente estuarino, con influencia mareal (Bady, Rivadeneira, \& Barragán, 2014).

$\mathrm{T}$, la arenisca $\mathrm{T}$ fue depositada luego de una importante regresión marina con un desplazamiento de la línea de costa en dirección Oeste, a partir de la cual se desarrollaron ambientes estuarinos con ingresos fluviales afectados por mareas, canales mareales, a los que se les asigna como areniscas inferiores y facies menos desarrolladas de barras, litofacies de llanura lodosa y areniscas glauconíticas de plataforma marina somera, dentro del cortejo netamente transgresivo de las areniscas definidas como superiores. (Bady, Rivadeneira, \& Barragán, 2014).

$\mathbf{U}$, el resultado de caídas en el nivel del mar, que dieron lugar a valles socavados, sobre los que se depositaron secuencias arenosas generalmente retrogradantes, producto de episodios transgresivos, que evolucionan desde un ambiente fluvial-estuarino (Bady, Rivadeneira, \& Barragán, 2014).

Basal Tena, se describen rellenos de canal con clastos gruesos, conglomeráticos a la base, posiblemente de origen fluvial. En partes se observa influencia mareal (Bady, Rivadeneira, \& Barragán, 2014).
Los ambientes sedimentarios de las arenas de interés del Campo X se detallan en la Tabla 1.

Tabla 1. Ambientes sedimentarios del campo en estudio. (Almeida \& Cárdenas, 2010).

\begin{tabular}{|l|c|c|c|c|}
\hline \multicolumn{5}{|c|}{ Ambientes de depositación del campo en estudio } \\
\cline { 1 - 4 } Formación & \multirow{2}{*}{ Hollín } & $\mathrm{T}$ & $\mathrm{U}$ & Basal Tena \\
\cline { 1 - 4 } Ambiente & $\mathrm{x}$ & $\mathrm{x}$ & $\mathrm{x}$ & $\mathrm{x}$ \\
\hline Fluvial & $\mathrm{x}$ & $\mathrm{x}$ & $\mathrm{x}$ & $\mathrm{x}$ \\
\hline Carbonatado & & $\mathrm{x}$ & $\mathrm{x}$ & \\
\hline Turbidítico & & & & \\
\hline
\end{tabular}

\subsubsection{Descripción estructural}

\subsubsection{Fallas}

El campo en estudio presenta fallas que se han descubierto entre los anticlinales que existen en la cuenca, describiendo una trayectoria noreste - sureste, las fallas son de tipo subverticales inversas que se presume es causada por el periodo cretácico por el alto ángulo de deformación producidas por fuerzas compresionales propias de la zona andina en que se encuentra (Acosta, 2016).

\subsubsection{Trampas}

El Campo en estudio es un anticlinal asimétrico elongado de relieve suave con orientación norte-sur, producido por un levantamiento del basamento tal como se muestra en la Figura 4.(Acosta, 2016).

\subsection{Estratigrafía de los pozos en estudio}

En la Tabla 6, del Anexo 1, se observa una correlación de los topes de las formaciones litológicas de los 5 pozos analizados, la caracterización de la geología brinda una ventaja al equipo de exploración sobre todo a la hora de diseñar los fluidos de perforación.

$\mathrm{Al}$ analizar los datos se aprecia la correspondencia que guardan las formaciones en todos los pozos de estudio, existe uniformidad en la profundidad de los mismos, así se demuestra como en una misma zona las distintas formaciones cubren el área de la Cuenca como una alfombra, con una ligera variación en la profundidad y un cambio en la potencia de los estratos.

Conocer la profundidad de los estratos en el Campo en estudio permite anticiparse en futuras perforaciones a tomar medidas correctivas frente a eventualidades en formaciones problemáticas, también ayuda a optimizar los parámetros de perforación en base de las lecciones aprendidas de los pozos analizados. 
Se aprecia que la formación Hollín (objetivo principal de los 5 pozos) aparece a una profundidad promedio de 8900 pies. Esta información fue recabada de los informes finales de perforación específicamente de los reportes litológicos, los datos en los reportes se lo hacen a partir de la formación Orteguaza.

\subsection{Procesamiento de datos obtenidos de herramientas de sondeo}

La muestra de 5 pozos pertenece a una misma locación, los sondeos fueron corridos como parte de los estudios complementarios para el desarrollo del campo, el análisis se lo realiza con el sondeo correspondiente al Pozo 2, el cual es presentado en este trabajo.

\subsubsection{Reconocimiento de marcadores regionales}

El análisis del sondeo se inicia determinando los marcadores regionales, los cuales son delimitadores naturales entre la finalización de una formación y el inicio de otra; estos marcadores son carbonos también denominados duros y calcáreos, los cuales se caracterizan por mostrar una variación marcada en el registro de Rayos Gamma (un pico).

En la Cuenca Oriente existen tres formaciones de interés en orden descendente Tena, Napo y Hollín. (Díaz, 2007). Las lecturas son aproximadas y están en pies MD.

La disposición de los marcadores regionales permite identificar y diferenciar las formaciones de interés puesto que actúa como un separador natural, la tabla 2 presenta la localización de los marcadores y las formaciones.

Tabla 2. Marcadores regionales.

\begin{tabular}{|c|c|}
\hline \multicolumn{2}{|c|}{ Marcadores regionales } \\
Formación & Observación \\
\hline Tena & \multirow{2}{*}{$\begin{array}{c}\text { El marcador "L" se coloca entre } \\
\text { las formaciones Tena y Napo }\end{array}$} \\
\cline { 1 - 1 } Marcador regional L & $\begin{array}{c}\text { El marcador "C" se coloca entre } \\
\text { las formaciones Napo y Hollín }\end{array}$ \\
\hline Marcador regional C
\end{tabular}

\subsubsection{Reconocimiento de marcadores regionales.}

El Gamma Ray permite determinar la presencia de los picos característicos de estos marcadores (carbonatos), lo que se confirma con un incremento en el registro de densidad con valores próximos a los de formaciones calcáreas de $2.70 \mathrm{gr} / \mathrm{cc}$ que los localiza a una profundidad de $9108 \mathrm{ft}$ (MD) lo que define al marcador regional L, y a una profundidad de $1014 \mathrm{ft}$ (MD) el marcador regional $\mathrm{C}$, como ejemplo se adjunta la ubicación del marcador L en el registro eléctrico de la figura 1 que corresponde al Pozo 2 .

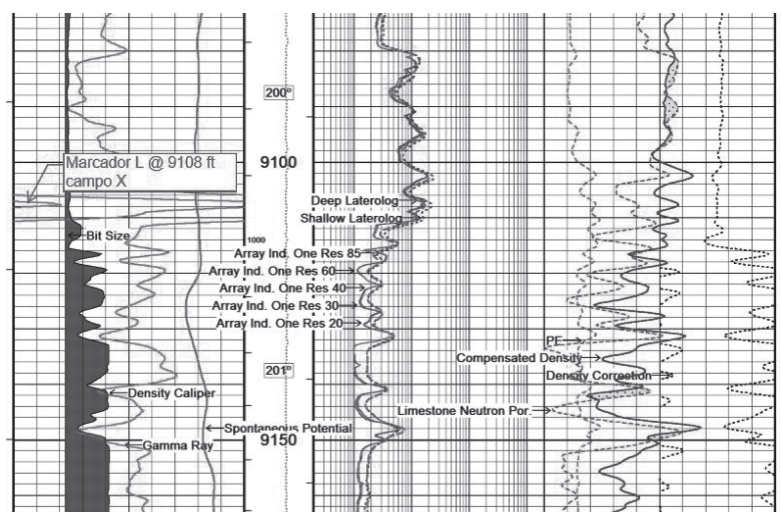

Figura 1. Marcador regional L del pozo 2.

\subsubsection{Determinación de las zonas de interés}

A través de los registros eléctricos se localizaron las arenas productoras de esta zona, se especifica el espesor de las mismas y se detallan algunas de las lecturas encontradas.

\subsubsection{Formación Basal Tena}

Tomando como referencia el marcador regional L (Figura 1) observando el registro en el sentido de fondo - superficie, se encuentra la formación Basal Tena. El Gamma Ray de $8892 \mathrm{ft}$ a $8908 \mathrm{ft}$ (14 pies de espesor) muestra una alteración, lo cual guarda correspondencia con los registros de densidad, resistividad y neutrón, en el Gamma Ray da un valor bajo de 40 API y se mantiene regular lo que hace referencia a una arena limpia. Las curvas de porosidad se cruzan en el mismo intervalo de 14 pies, la resistividad es de $60 \Omega \mathrm{m}$, por lo anterior se puede concluir que se identifica una zona permeable y porosa, lo cual confirma la presencia del reservorio Basal Tena.

\subsubsection{Formación U superior}

Tomando como referencia el límite natural entre la Formación Tena y la Formación Napo (marcador regional L), al avanzar en profundidad se localiza a la arena $\mathrm{U}$ superior la cual pertenece a la formación Napo. La arena U superior se identifica de $9532 \mathrm{ft}$ a $9556 \mathrm{ft}$ con 24 pies de espesor. El sondeo al llegar a esta arena presenta un cambio notable en el Gamma Ray lo que indica una transición litológica, esto guarda correspondencia con los registros de densidad, resistividad y neutrón, el Gamma Ray da una lectura aproximada de 90 API, este indicador se muestra 
bastante irregular por la presencia de incrustaciones de arcilla (alta radiación natural). Las curvas de porosidad no se cruzan en el intervalo de 24 pies, la resistividad tiene un valor de $50 \Omega \mathrm{m}$, por lo anterior se puede concluir que se identifica una zona que probablemente sea impermeable con presencia de lutitas. El caliper indica que en la zona existen pequeños derrumbes característico de formaciones que contiene arcilla.

\subsubsection{Formación $T$ inferior}

Esta sección se extiende desde los $9842 \mathrm{ft}$ hasta los $9886 \mathrm{ft}$ (espesor de 44 pies), se puede observar que el Gamma Ray da 100 API, en el centro del estrato se identifica un pico de 130 API, este indicador muestra claramente la intercalación de una arcilla, (valores irregulares, alta radiación natural). Las curvas de porosidad no se cruzan en el intervalo donde se registra el pico en el Gamma Ray, por el contrario, estas mismas curvas se cruzan donde los valores de Gamma Ray son bajos, la resistividad es de $10 \Omega \mathrm{m}$ en la zona arcillosa y de $80 \Omega \mathrm{m}$ en la zona arenosa, por lo anterior se puede concluir que se identifica una zona permeable y porosa (intercalada con lutita), el registro caliper también evidencia la formación de revoque lo que corrobora la presencia de una formación con buena porosidad, se confirma la presencia del reservorio $\mathrm{T}$ inferior.

\subsubsection{Formación Hollín superior}

Tomando como referencia el límite natural entre la formación Napo y Hollín (marcador regional C, ver figura 2), al avanzar un poco en profundidad se localiza a la arena Hollín Superior de $10042 \mathrm{ft} \mathrm{a} 10024 \mathrm{ft}$ con 26 pies de espesor (Figura 2). El sondeo presenta un cambio notable en el Gamma Ray lo que indica una transición litológica que guarda correspondencia con los registros de densidad, resistividad y neutrón, al llegar a esta zona el Gamma Ray presenta valores medios (90 API), altos (120 API) y bajos (40 API) respectivamente, este marcador es irregular. Las curvas de porosidad no se cruzan en el intervalo de 24 pies, la resistividad tiene un valor que se aproxima a $100 \Omega \mathrm{m}$, por lo anterior se puede concluir que se identifica una zona que probablemente sea impermeable con presencia de lutitas desde los 10042 $\mathrm{ft}$ hasta $10018 \mathrm{ft}$, también se observa la presencia de arenas al final del estrato de $10018 \mathrm{ft}$ a $10024 \mathrm{ft}$. El caliper indica un revoque que apenas se lo puede distinguir en la zona de donde el Gamma Ray tiene valores bajos, todo lo anteriormente descrito se puede observar en la Figura 2.

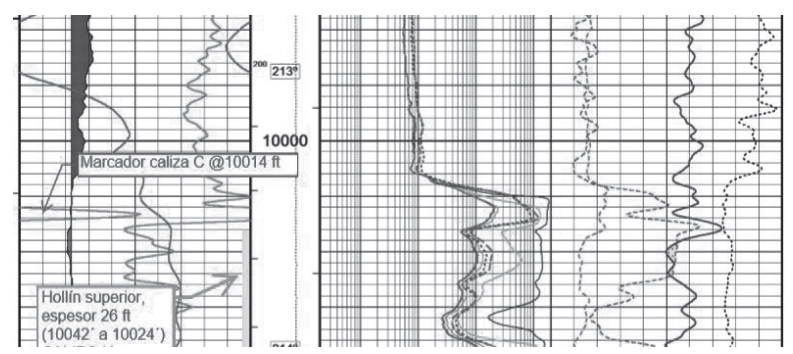

Figura 2. Formación Hollín superior del pozo 2.

\subsubsection{Formación Hollín inferior}

Los pozos analizados tienen como objetivo esta formación (ver Figura 2). Al observar el registro, fondo - superficie, se encuentra la formación Hollín Superior. El Gamma Ray de 10086 ft a 10150 ft (64 pies de espesor) muestra una alteración que guarda correspondencia con los registros de densidad, resistividad y neutrón, en el Gamma Ray da 20 API y se mantiene regular lo que hace referencia a una arena limpia. Las curvas de porosidad se cruzan en el mismo intervalo de 64 pies, la resistividad tiene un valor pico al inicio de la formación de $400 \Omega \mathrm{m}$, pero el mismo se estabiliza en $100 \Omega \mathrm{m}$, por lo anterior se puede concluir que se identifica una zona permeable y porosa, el registro caliper indica la formación de revoque lo cual confirma la presencia del reservorio Hollín inferior (Figura 3).

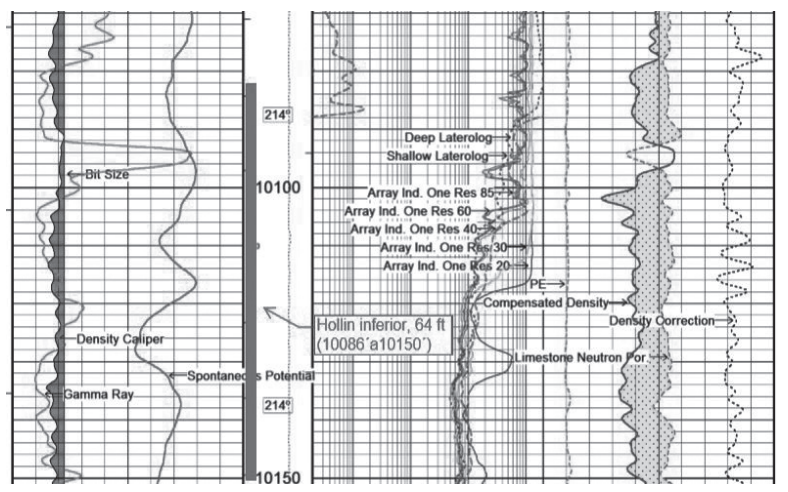

Figura 3. Formación Hollín inferior pozo 2.

\subsubsection{Determinación de las zonas inestables}

Determinar las formaciones arcillosas, fracturadas o inconsolidadas mediante la interpretación de los sondeos permitirá tomar medidas y correctivos necesarios al llegar a estas formaciones, estos contingentes operativos deben estar contemplados en el planeamiento de futuros pozos. Los resultados se resumen en la Tabla 7 , del Anexo 1. 


\subsection{Factores geomecánicos del campo en estudio}

En perforación es importante determinar la orientación de los esfuerzos principales en la cara del pozo, orientación y magnitud de los esfuerzos de la formación y la orientación del pozo. Al considerar estas variables se puede mejorar la selección de trayectoria del pozo a perforar, provocar el menor daño a las capas a perforarse, atravesarlas con el ángulo más adecuado $\mathrm{y}$ que presenten menor resistencia obteniendo buenas ratas de perforación.

En el diseño del hoyo se debería considerar que la dirección de los pozos sea transversal a la orientación de los esfuerzos regionales de la formación puesto que son los causantes de la configuración de los anticlinales, es decir que el pozo debería tener una trayectoria casi paralela a la dirección aparente de las capas del anticlinal, por cuanto se ha asociado que los pozos con mayores ratas de perforación obedecen a este parámetro de diseño (Almeida \& Cárdenas, 2010).

Partiendo de la premisa que el anticlinal del campo en estudio tiene una orientación $\mathrm{N}-\mathrm{S}$ y los esfuerzos regionales de formación tienen una orientación W-E, se podrá establecer una relación entre la dirección del anticlinal y la de los pozos en estudio para determinar aquellos que siguen una dirección transversal.

En la Figura 4 se observa la orientación de los pozos respecto al anticlinal y la tabla 3 indica mediante colores un rango de ángulos de la disposición (ubicación) de los pozos respecto del anticlinal:
Verde, representa una orientación transversal (trayectoria óptima).

Amarillo, representa una orientación diagonal (trayectoria a mejorar).

Rojo, representa una orientación paralela (trayectoria que se debe corregir).

La observación experimental establece una relación entre los esfuerzos de las deformaciones durante el proceso de carga y rotura de la roca, está claro que la resistencia de los macizos rocosos es función de la resistencia de la matriz rocosa y de las discontinuidades (ambas extremadamente variables) (Calla, 2014) sin embargo la orientación perpendicular entre los esfuerzos de formación y la dirección del pozo favorecen a la hora de incrementar la ROP.

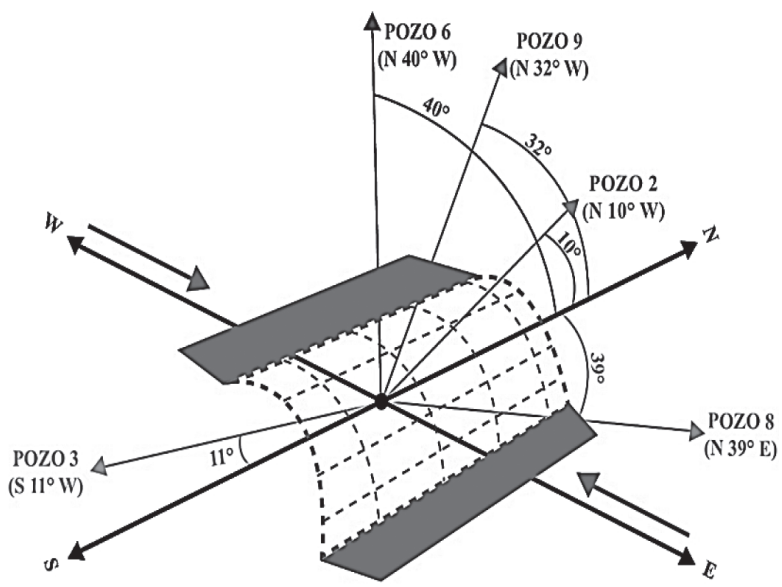

Figura 4. Esfuerzos regionales del anticlinal de la zona.

Tabla 3. Orientación de los pozos.

\begin{tabular}{|c|c|c|c|c|c|}
\hline \multirow{2}{*}{ Pozo } & \multirow{2}{*}{ Dirección } & Indicador & Transversal & Diagonal & Diagonal a paralelo \\
\hline & & Posición esfuerzos vs. dirección pozo & $0^{\circ}$ a $15^{\circ}$ & $16^{\circ}$ a $30^{\circ}$ & $>31^{\circ}$ \\
\hline 2 & $\mathrm{~N} 10^{\circ} \mathrm{W}$ & Transversal & & & \\
\hline 3 & $\mathrm{~S} 11^{\circ} \mathrm{W}$ & Transversal & & & \\
\hline 6 & $\mathrm{~N} 40^{\circ} \mathrm{W}$ & Diagonal a $\sim$ paralelo & & & \\
\hline 8 & $\mathrm{~N} 39^{\circ} \mathrm{E}$ & Diagonal a $\sim$ paralelo & & & \\
\hline 9 & $\mathrm{~N} 32^{\circ} \mathrm{W}$ & Diagonal a $\sim$ paralelo & & & \\
\hline \multicolumn{2}{|c|}{ Orientación del anticlinal } & $\mathrm{N}-\mathrm{S}$ & \multicolumn{2}{|c|}{ Orientación esfuerzos principales } & $\mathrm{W}-\mathrm{E}$ \\
\hline \multirow{3}{*}{\multicolumn{3}{|c|}{ Contraste de ROP considerando la orientación de los pozos }} & Sección & Pozo 2 & Pozo 9 \\
\hline & & & $161 / 4 ”$ & $70.9 \mathrm{ft} / \mathrm{h}$ & $16.5 \mathrm{ft} / \mathrm{h}$ \\
\hline & & & $121 / 2 ”$ & $31 \mathrm{ft} / \mathrm{h}$ & $29.7 \mathrm{ft} / \mathrm{h}$ \\
\hline
\end{tabular}




\subsection{Problemas asociados a la litología del campo en estudio}

La Tabla 4, indica los problemas relacionados a la sección perforada asociando el NPT a la litología.

La pega de tubería en las secciones de $8 \frac{1}{2}$ " (61 horas) y de $121 / 4$ " (5.5 horas) es uno de los problemas que se debe anticipar y la pesca en la sección de 6 1/8" (267 horas) requieren ser contempladas en la planificación para mejorar la respuesta ante estos problemas. La Figura 5 , expone los NPT por sección asociados a la litología.

Tabla 4. NPT por sección asociado a la litología.

\begin{tabular}{|c|c|c|c|c|c|}
\hline \multicolumn{6}{|c|}{ NPT (hrs) por sección asociado a la litología } \\
\hline $\begin{array}{c}\text { Problemas asociados } \\
\text { a la litología }\end{array}$ & $26 ”$ & $16 ”$ & $121 / 2$ “ & $81 / 2$ “ & $61 / 8$ “ \\
\hline Pega de tubería & 0 & 0 & 5.5 & 60 & 0 \\
\hline Pesca & 0 & 0 & 0 & 0 & 266.5 \\
\hline Pérdida de circulación & 0 & 0 & 0 & 0 & 0 \\
\hline Arremetidas & 0 & 4.5 & 0 & 0 & 0 \\
\hline
\end{tabular}

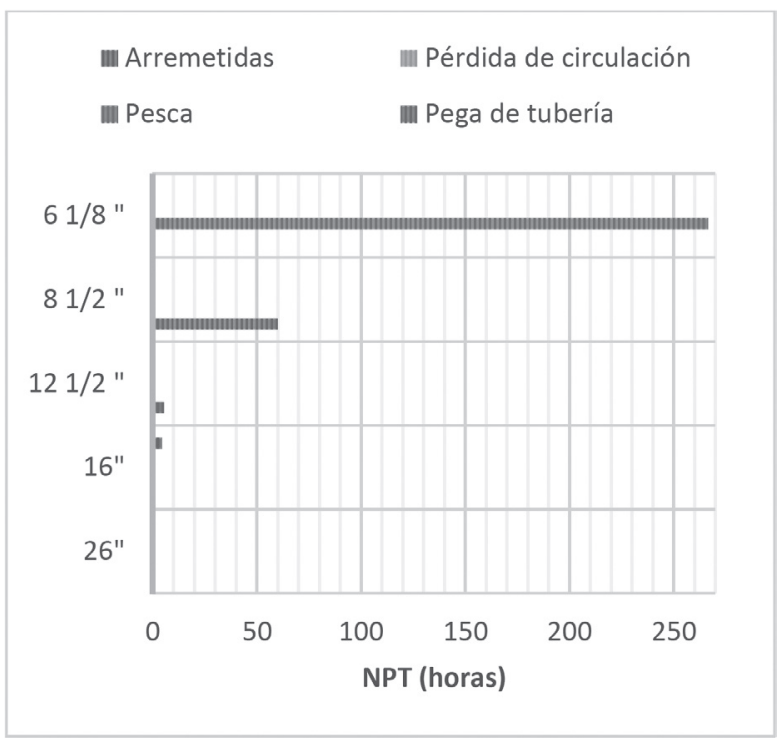

Figura 5. NPT asociados a la litología.

\section{Propuesta del pozo tipo}

\subsection{Parámetros de perforación}

Al contrastar las variables de perforación se determina el desempeño de los pozos, en este punto se dejará la parte operativa de lado para centrar la atención únicamente en los valores que se adaptaron mejor a las condiciones regionales y como resultado de esto reflejan un incremento en la tasa de perforación. Los pozos 3,6,9 y 2,8 son de 5 y 4 secciones respectivamente y todos son pozos horizontales.

Los parámetros de perforación por cada sección que presentan un desempeño óptimo se los clasifica y se realizará una modelo con aquellas variables que en condiciones de operaciones reales incrementaron la ROP, dichos parámetros construirán sección a sección lo que denominaremos el pozo tipo.

Se seleccionaron los parámetros (densidad, caudal, WOB, RPM) que optimizaron el avance considerando el supuesto de que éstos a su vez son el reflejo de las mejores prácticas operativas en las cuales existe una reducción de las tareas no productivas, la tabla 5 , indica los parámetros que generan los ROP más elevados.

Tabla 5. Parámetros de perforación que optimizan la tasa de penetración.

\begin{tabular}{|c|c|c|c|c|c|c|c|}
\hline \multicolumn{7}{|c|}{ Pozo tipo, parámetros de perforación } & \multirow{3}{*}{\begin{tabular}{|c}
$\%$ \\
incremento \\
ROP \\
optimizada \\
vs. ROP \\
más lenta \\
80
\end{tabular}} \\
\hline Sección & \multicolumn{2}{|c|}{$\begin{array}{c}\text { Densidad } \\
\text { (lb /gal) }\end{array}$} & \multicolumn{2}{|c|}{$\begin{array}{c}\text { Caudal } \\
\text { GPM }\end{array}$} & \multirow{2}{*}{\begin{tabular}{|l} 
WOB \\
(Klb)
\end{tabular}} & \multirow{2}{*}{\begin{tabular}{|l|} 
RPM \\
$26-120$ \\
\end{tabular}} & \\
\hline $26 "$ & 8.4 & 9.5 & 230 & 230 & & & \\
\hline $16 "$ & 9.9 & 13.8 & 400 & 1000 & $20-30$ & $40-63$ & 80 \\
\hline $121 / 4^{\prime \prime}$ & 9.9 & 12.2 & 700 & 850 & $15-20$ & $120-150$ & 15 \\
\hline $81 / 2 "$ & 8.9 & 9.2 & 400 & 450 & 14-18 & 120 & 59 \\
\hline $61 / 8 ”$ & 8.9 & 9.3 & 240 & 270 & $6-10$ & $60-120$ & 42 \\
\hline
\end{tabular}

\subsection{Curva de densidades del lodo propuesto}

Se realiza la propuesta de densidades como resultado de analizar los reportes de los lodos de perforación de las secciones con la tasa de penetración más elevada, y con el resultado se construye la curva del pozo tipo, la misma que se detalla en la figura 9 del Anexo 1.

La curva propuesta es el resultado de una mejor adaptación de las densidades del lodo a las formaciones geológicas atravesadas. Con esto se asegura que el fluido seleccionado se comporta de mejor forma ante las formaciones inestables (estabiliza el pozo no reaming) y tiene el peso ideal cuando está a la profundidad de las formaciones permeables (evitando fracturarlas o impidiendo la formación de un excesivo revoque). 
Al optimizar la tasa de penetración reduciremos el contacto del lodo de perforación con las formaciones de interés lo que permite entregar un pozo en excelentes condiciones para la producción, evitando así el incremento del radio de invasión y por ende un potencial incremento en el factor de skin.

\subsection{Selección de trayectoria óptima}

A partir de los esfuerzos principales determinados en la pared del pozo y su consecuente diagrama de estabilidad, los cuales se interpretan según lo indicado en el estudio de Geomecánica de Liliana Páramo, documento que también describe las características de un diagrama de estabilidad polar, en donde el color rojo representa orientaciones de pozo relativamente inestables que requieren un mayor peso de lodo para evitar la iniciación de breakouts, mientras que el color azul representa orientaciones de pozo relativamente estables en las que se requieren pesos de lodo más bajos para evitar la iniciación de los breakouts (Páramo, 2016). Se realizó la interpretación de los diagramas para determinar una trayectoria óptima.
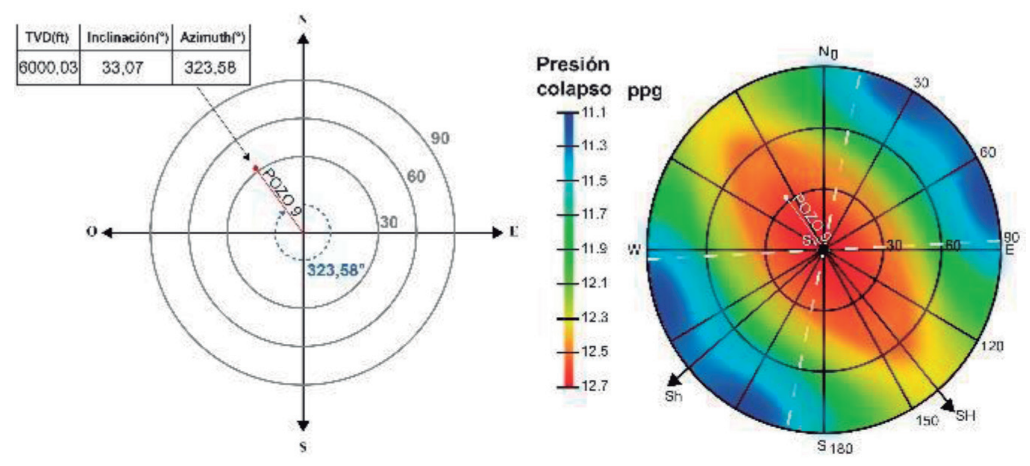

Figura 6. Diagrama de estabilidad a $6000 \mathrm{ft}$.

A 7000 pies la orientación del pozo 9 se orienta en una zona inestable en direcciona hacia un flanco que requiere un mayor peso de lodo para evitar el inicio de breakouts, está en una zona inestable, se recomienda

\subsubsection{Considerando la variación}

de los esfuerzos principales

Una de las formas de conseguir estabilidad en el pozo se logra perforando en la dirección de máxima tensión horizontal. Cuando estos pozos se fracturan hidráulicamente, la fractura crece a lo largo del pozo en la dirección del azimut del pozo. La perforación hacia la tensión horizontal mínima ( $\sigma \mathrm{min})$ es un desafío de integridad y estabilidad del pozo, esto se debe a que el pozo está sujeto a mayores tensiones y por lo tanto requiere una mayor densidad del lodo para controlar el colapso de la formación. (Zillur, Adnan , Al- Anazi, \& Mahbub, 2012).

\subsubsection{Considerando diagramas polares de estabilidad}

A 6000 pies la orientación del pozo 9 no es la adecuada, se direcciona hacia un flanco que requiere un mayor peso de lodo, está en una zona inestable, se recomienda un Azimut entre $0^{\circ}$ y $90^{\circ}$ o entre $180^{\circ}$ y $270^{\circ}$. La figura 6 representa la orientación del pozo 9 dentro del diagrama. un Azimut entre $10^{\circ}-40^{\circ}$ y $55^{\circ}-90^{\circ}$ o entre $190^{\circ}-235^{\circ}$ y $250^{\circ}-270^{\circ}$. La figura 7 representa la orientación del pozo 9 dentro del diagrama.
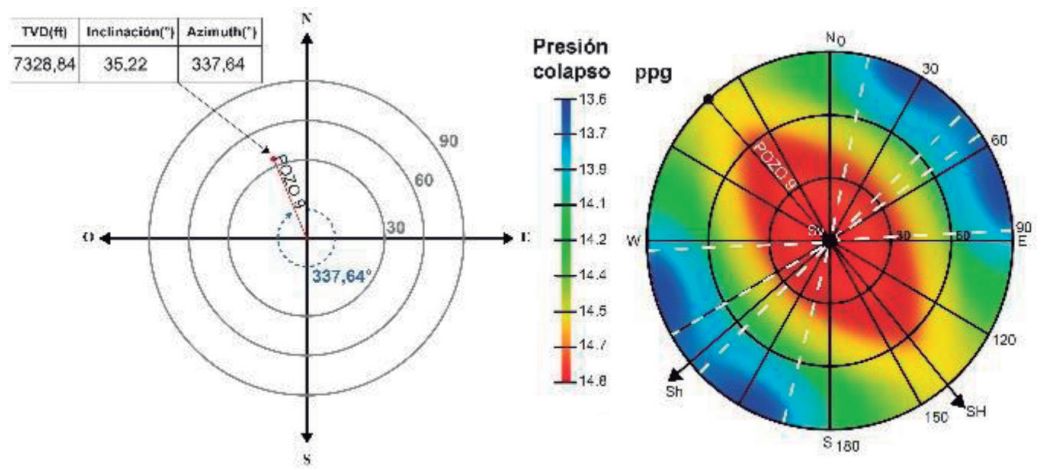

Figura 7. Diagrama de estabilidad a $7000 \mathrm{ft}$. 
A 9000 pies la orientación del pozo 9 se sitúa en una orientación que se puede mejorar, se direcciona hacia un flanco que requiere un mayor peso de lodo, está en una zona inestable, se recomienda un Azimut entre $0^{\circ}-30^{\circ}$ y $60-90^{\circ}$ o entre $190^{\circ}-220^{\circ}$ y $240^{\circ}-270^{\circ}$. La figura 8 representa la orientación del pozo.

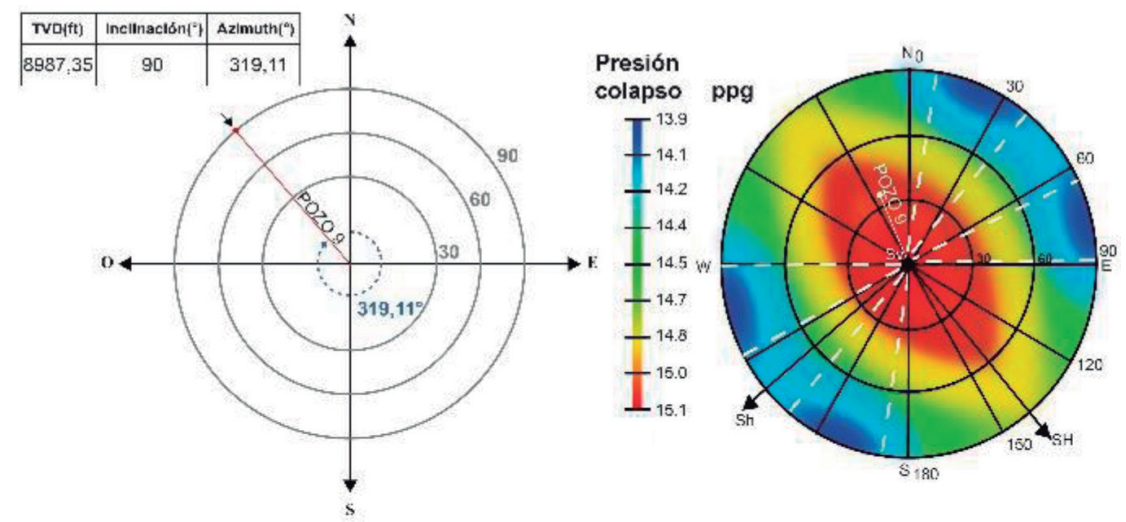

Figura 8. Diagrama de estabilidad a $9000 \mathrm{ft}$.

\section{Conclusiones}

Uno de los factores que afecta la perforabilidad en las arenas de interés son los ambientes de depositación, en el campo de estudio se identificaron los siguientes: Fluvial, Carbonatado y Turbidítico.

En los pozos 2 y 3 los esfuerzos de formación del anticlinal y la dirección del pozo se disponen de forma tal que tienen una orientación perpendicular, lo que favorece a la hora de incrementar la ROP, los pozos 6,8 y 9 sus esfuerzos se orientan entre sí de forma paralela la cual limita el incremento de la ROP.

Los diagramas de estabilidad revelan que se puede tomar en consideración otras orientaciones. El análisis a $6000 \mathrm{ft}, 7000 \mathrm{ft}$ y $9000 \mathrm{ft}$ indica que el pozo posee una trayectoria que lo ubica en la zona que requiere un mayor peso de lodo para lograr la estabilidad. Esto quiere decir que el pozo lleva una dirección contraria a la tensión horizontal máxima, lo cual se verifica en las figuras 6,7 y 8 .

Después de realizar una correlación mediante los registros litológicos para determinar las formaciones atravesadas al perforar, se puede determinar las profundidades de las zonas, así como la potencia de las arenas de interés.

Los registros eléctricos permitieron caracterizar las zonas problemáticas, determinar el espesor de las mismas y se detallaron algunas de las lecturas de los sondeos de dichas zonas, la presencia de arcillas es el denominador común en estas formaciones.
Se identificó el tope y las bases de las formaciones productoras mediante los registros eléctricos, su posición fue corroborada con los registros litológicos.

Se identificaron en los 5 pozos los problemas que tienen como raíz la litología, estos son una arremetida (4.5 horas) en la sección de 16", pega de tubería (65.5 horas) presentes en la sección de 12 1/4 “ y 8 1/2 “, también tenemos un problema aislado en de pesca (266.5 horas) en la sección de 6 1/8".

Se determinaron los parámetros de perforación que en cada sección de los 5 pozos analizados ofrecieron un mejor desempeño de la ROP, los valores se detallan en la tabla 5 y son las variables que se utilizan en el pozo tipo para incrementar la tasa de penetración. Al contrastar la tasa de penetración de las secciones perforadas entre aquellas que hacen uso de los parámetros optimizados versus las que no, se evidencia hasta un incremento del $80 \%$ en la tasa de perforación, la tabla 5 expone el incremento que se podría alcanzar.

Tomando en consideración el Mud Weight que genera la ROP más alta en cada sección se realizó la curva de densidades del lodo para el pozo tipo, como resultado se obtiene una curva adaptada a las formaciones geológicas atravesada, en la figura 9 del Anexo 1 se expone la curva de densedades.

\section{Referencias}

1. Acosta, A. (2016). Análisis de la Producción de Agua en los Pozos Horizontales del Campo Oso. Quito: Universidad Tecnológica Equinoccial. 
2. Almeida , D., \& Cárdenas, D. (2010). Optimización de la Perforación de Pozos Direccionales de los Principales Campos de Petroproducción en la Cuenca Oriente en Base de la Caracterización Litológica. Quito: Escuela Politécnica Nacional.

3. Ayala, D., Benítez, A., \& Valencia, R. (2017). Optimización de la Tasa de Penetración mediante el análisis de las vibraciones al perforar, caso de estudio Ecuador. Revista Fuentes, 15(1), 27-40.

4. Ayala, D., Torres, H., Valencia, R., \& Loaiza, M. (2016). Impacto del Tiempo no Productivo en operaciones de perforación y análisis de los datos mediante la prueba de Chicuadrado. Revista Fuentes, 14(2), 5-18.

5. Ayala, F. E. B., Gómez, J. Q., \& León, E. A. (2011). Estudio de factibilidad del uso del biodiesel como fase contínua en lodos de perforación de emulsión inversa. Revista Fuentes, 9(1).

6. Bady, P., Rivadeneira, M., \& Barragán, R. (2014). La Cuenca Oriente Geología y Petróleo. Quito: Instituto Frances del Petróleo.

7. Calla, J. (2014). Mecánica de Rocas, Resistencia y Deformabilidad. Mexico: Slideshare.

8. Campana, D. E. A., \& Tapia, R. A. V. (2017). Evaluación cualitativa de la limpieza de hoyo en pozos de alta inclinación-alto desplazamiento en la Cuenca Oriente. Revista Fuentes, 15(2), 49-56.

9. Díaz, C. (2007). Alcance de los Registros Acústicos de Última Tecnología en Aplicaciones: Petrofísicas, Geológicas y Geomecánicas de los Pozos Perforados en el período 2002 - 2004 en la Cuenca Oriente Ecuatoriana. Quito: Escuela Politécnica Nacional.

10. Garnier, A., \& Van Lingen, N. (1959). Phenomena Affecting Drilling Rates at Depth, SPE 1097 G. Houston: SPE.

11. Graham, A., \& Muench, N. (1959). Analytical determination of optimun bit weight and rotary speed combintions. SPE 1349 G. Dallas: SPE.

12. Hawker, D., Vogt, K., \& Robinson, A. (2001). DATALOG Manual de Operaciones en el Pozo.
Alberta: DATALOG.

13. Loaiza, M., Ayala, D., Torres, H., \& Ayala, S. (2018). Tiempo no productivo en pozos de dos secciones, caso de estudio Ecuador. Fuentes: El reventón energético, 16(1), 7-17.

14. Medina, C. A. C., Martínez, J. J. S., León, E. A., \& Boada, W. M. (2013). Análisis reológico para predecir y mejorar el comportamiento hidráulico durante la perforación de un pozo. Revista Fuentes, 11(1).

15. Montes, A., Carreño, W., \& Guío, M. (2018). Aspectos de la perforación de pozos complejos en piedemonte en tiempos de crisis. Revista Fuentes, 16(1), 87-97.

16. Ortiz-Vargas, D. A., Carrillo, Z. H. C., \& Tarazona, D. M. (2018). Modelo matemático para determinar presión permitida de pérdidas de filtrado de lodo controladas. Fuentes: El reventón energético, 16(1), 47-55.

17. Páramo, L. (2016). Análisis Geomecánico y de Estabilidad para el pozo \#3 del Campo Caso Estudio. Medellín: Universidad Nacional De Colombia.

18. Portilla, H. E., Suárez, D. F., \& Corzo, R. (2012). Metodología para la optimización de parámetros de perforación a partir de propiedades geomecánicas. Revista Fuentes, $10(2)$.

19. Rashidi, B., \& Hareland, G. (2009). Lithological determination from logs. Calgary: DOI.

20. Saleem, Q., \& Tunio, N. (2011). Is It Possible to Ignore Problems Rissing During Vertical Drilling . Houston: Appl. Sci. Eng. Tecnol.

21. Solberg, S. (2012). Improved Drilling Process Through the determination of hardness and lithology boundaries. Trondheim: Norwegian University of Sciences and Technology.

22. Tuna, E. (2010). real Time Optimization of Drilling Parameters During Drilling Operations. Turquía: Middle East Technical University.

23. Zillur, R., Adnan , A.-K., Al- Anazi, H., \& Mahbub, A. (2012). Succesful drilling of lateral wells in minimun horizontal stress direction for optimal fracture placement. Dhahran: Saudi Aramco.

Recepción: 13 de noviembre de 2018

Aceptación: 30 de junio de 2019 


\section{Anexo 1}

Tabla 6. Registro litológico elaborado a partir de reportes de perforación.

\begin{tabular}{|c|c|c|c|c|c|}
\hline Formación & $\begin{array}{c}\text { Pozo } 2 \\
\text { (TVD) } \mathrm{ft}\end{array}$ & $\begin{array}{c}\text { Pozo } 3 \\
\text { (TVD) } \mathrm{ft}\end{array}$ & $\begin{array}{c}\text { Pozo } 6 \\
\text { (TVD) ft }\end{array}$ & $\begin{array}{c}\text { Pozo } 8 \\
\text { (TVD) } \mathrm{ft}\end{array}$ & $\begin{array}{c}\text { Pozo } 9 \\
\text { (TVD) } \mathrm{ft}\end{array}$ \\
\hline \multicolumn{6}{|l|}{ Mera } \\
\hline \multicolumn{6}{|l|}{ Mesa } \\
\hline \multicolumn{6}{|l|}{ Chambira } \\
\hline \multicolumn{6}{|l|}{ Arajuno } \\
\hline \multicolumn{6}{|l|}{ Chalcana } \\
\hline Orteguaza & 5036 & & & 5052 & 4998 \\
\hline Tiyuyacu & 5819 & 5825 & 5805 & 5874 & 5839 \\
\hline \multicolumn{6}{|c|}{ Conglomerado Superior Tiyuyacu } \\
\hline \multicolumn{6}{|c|}{ Conglomerado Inferior Tiyuyacu } \\
\hline Tena & 6883 & 6866 & 6851 & 6908 & 6875 \\
\hline Arenisca Basal Tena & 7873 & 7848 & 7852 & 7899 & 7858 \\
\hline Napo & 7877 & 7871 & 7860 & 7907 & 7869 \\
\hline Napo Basal & & 8801 & & & \\
\hline Caliza M-1 & 7911 & 7916 & 7908 & 7967 & 7915 \\
\hline \multicolumn{6}{|l|}{ Base Caliza M-1 } \\
\hline Caliza M-2 & 8216 & 8160 & 8151 & 8206 & 8172 \\
\hline \multicolumn{6}{|l|}{ Base Caliza M-2 } \\
\hline Zona Caliza A & 8364 & 8323 & 8314 & 8361 & 8325 \\
\hline Arena U superior & 8540 & 8485 & 8473 & 8531 & 8483 \\
\hline \multicolumn{6}{|l|}{ Arenisca U Inferior } \\
\hline Arena U principal & 8580 & 8521 & 8514 & 8558 & 8534 \\
\hline \multicolumn{6}{|l|}{ Base Arenisca U inferior } \\
\hline Lutita Napo Media & 8609 & & 8540 & 8593 & 8563 \\
\hline \multicolumn{6}{|l|}{ Lutita B } \\
\hline Caliza B & 8717 & 8661 & 8657 & 8709 & 8667 \\
\hline Arenisca T Superior & 8727 & 8675 & 8669 & 8723 & 8677 \\
\hline \multicolumn{6}{|l|}{ Arenisca $\mathrm{T}$ inferior } \\
\hline Arenisca T Principal & 8781 & 8739 & 8719 & 8795 & 8725 \\
\hline Lutita Napo Inferior & 8781 & & 8804 & 8870 & 8806 \\
\hline Hollín Superior & 8843 & 8919 & 8905 & 8956 & 8911 \\
\hline Hollín Inferior & 8947 & & & & \\
\hline Hollín Principal & & & 8964 & 9019 & 8969 \\
\hline
\end{tabular}


Tabla 7. Análisis de zonas inestables identificadas a partir de registros eléctricos.

\begin{tabular}{|c|c|c|}
\hline Zona & MD (ft) & Comentario \\
\hline 1 & \begin{tabular}{l}
1 \\
\multirow{2}{*}{} \\
$\infty$ \\
$\infty$ \\
$\infty$
\end{tabular} & $\begin{array}{l}\text { Se encuentra inmediatamente después del zapato de asentamiento de la tubería de revestimiento, el gamma } \\
\text { ray presenta una lectura de } 120 \mathrm{API} \text { y una resistividad que inicia en } 4 \text { y avanza hasta } 10 \Omega \mathrm{m} \text {, el caliper } \\
\text { presenta la irregularidad del hoyo por sus derrumbes y un incremento en el diámetro que llega hasta } 14 \\
\text { pulgadas, el density registra valores estables en torno a } 2,45 \mathrm{gr} / \mathrm{cc} \text {, las lecturas indican la presencia de arcillas. }\end{array}$ \\
\hline 2 & 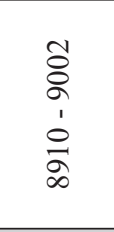 & $\begin{array}{l}\text { La segunda zona inestable presenta el gamma ray una lectura que se aproxima mucho a } 120 \text { API y se } \\
\text { mantiene con regularidad a lo largo de la zona descrita de } 92 \text { pies de espesor, la resistividad tiene una lectura } \\
\text { estable de entre } 3 \text { y } 4 \Omega \mathrm{m} \text {, el caliper presenta la irregularidad del hoyo por sus derrumbes y un incremento en } \\
\text { el diámetro que llega hasta } 14 \text { pulgadas, indicando una variación de la lectura normal de hasta } 2 \text { pies lo que } \\
\text { indica un derrumbe que se mantiene a lo largo de } 92 \text { pies, el density registra valores estables en torno a } 2,45 \\
\text { gr/cc las lecturas indican la presencia de arcillas. }\end{array}$ \\
\hline 3 & $\begin{array}{l}\text { 2े } \\
\text { సू } \\
1 \\
b \\
= \\
\bar{a}\end{array}$ & $\begin{array}{l}\text { La tercera zona se encuentra } 8 \text { pies por debajo del marcador regional "L" por esta razón las primeras lecturas } \\
\text { en el density son altas lo cual corresponde a la presencia de carbonatos (caliza), al descender en profundidad } \\
\text { el density fluctúa entre } 2,35-2,55 \mathrm{gr} / \mathrm{cc} \text {, el Gama Ray no es estable al inicio de esta zona influenciado por } \\
\text { los carbonatos y presenta valores altos este fluctúa entre los valores } 120 \text { y } 140 \text { API. La resistividad presenta } \\
\text { lecturas entre } 2-4 \Omega \mathrm{m} \text {, el caliper presenta un incremento de } 2 \text { pies lo cual muestra un derrumbe que se } \\
\text { mantiene a lo largo de } 180 \text { pies que confirma una sección inestable y por todas las lecturas indica ser una } \\
\text { formación de arcillas. }\end{array}$ \\
\hline 4 & 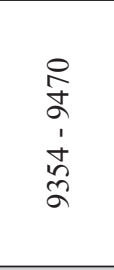 & $\begin{array}{l}\text { La cuarta zona indica lecturas irregulares en el density y fluctúa entre2,25- } 2,55 \text { gr/cc, el Gama Ray no es } \\
\text { estable pero se puede apreciar zonas donde la lectura se estabiliza de } 120 \text { a } 140 \text { API. La resistividad también } \\
\text { presenta irregularidad, pero guarda correspondencia con el gamma ray, en las zonas donde se estabiliza } \\
\text { presenta lecturas entre } 2 \text { - } 4 \Omega \mathrm{m} \text {, el caliper presenta un incremento de } 2 \text { pies lo que corresponde a un derrumbe } \\
\text { que se mantiene a lo largo de } 116 \text { pies, el cual al aumentar en profundidad el hoyo se acerca al diámetro de } \\
\text { calibre lo que se puede confirmar con el caliper, por lo anterior se menciona que esta sección es inestable y } \\
\text { por todas las lecturas indican una formación arcillosa. }\end{array}$ \\
\hline 5 & 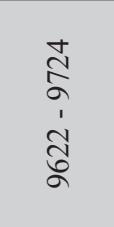 & $\begin{array}{l}\text { La quinta zona inestable presenta el gamma ray una lectura que se estabiliza en valores entre } 120 \text { y } 140 \\
\text { API y se mantiene con regularidad a lo largo de la zona descrita de } 102 \text { pies de espesor, la resistividad tiene } \\
\text { una lectura estable de entre } 4 \text { y } 5 \Omega \mathrm{m} \text {, el caliper presenta la irregularidad del hoyo por sus derrumbes y un } \\
\text { incremento en el diámetro, indicando una variación de la lectura normal de hasta } 2 \text { pies lo que corresponde a } \\
\text { un derrumbe que se mantiene a lo largo de } 102 \text { pies, el density se ubica en valores de } 2,45 \text { gr/cc las lecturas } \\
\text { indican la presencia de arcillas. }\end{array}$ \\
\hline
\end{tabular}

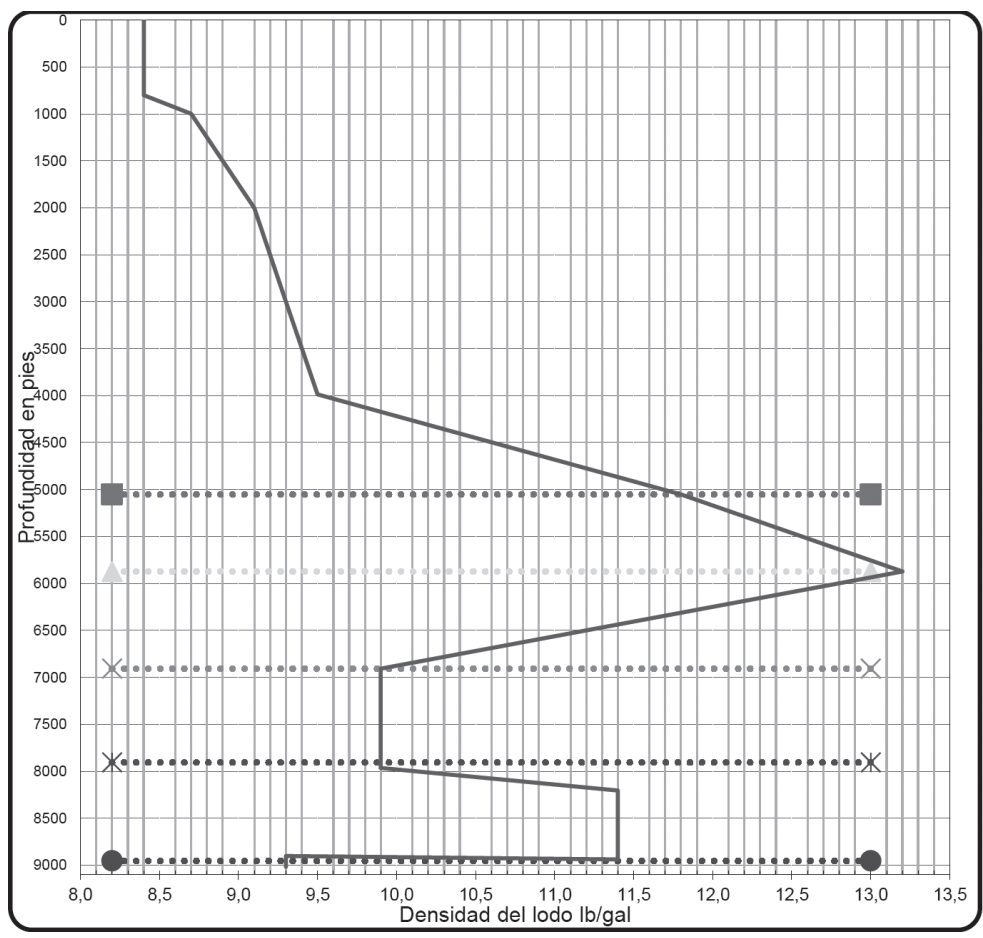

Figura 9. Diagrama de densidades del fluido de perforación del pozo tipo. 